\title{
The application of unified power flow controller in power system of dynamic power quality
}

\author{
jun Li, kuikui Zhang, tingjian Zhong, bin Chen and yiwen Hu \\ Jiangxi Vocational and Technical College of electricity, Nanchang ,330032 China \\ Email:jxdlztj@163.com
}

Keywords: flexible ac transmission system (FACTS); UPFC; power quality

\begin{abstract}
UPFC is one of the series-parallel combination FACTS devices, it has no additional storage or power equipment, but it can be simultaneously achieved active and reactive power compensation function, and also it can be realized to rapid dynamic adjustment in the entire power system, such as voltage , impedance, phase and power parameters, improving the stability of power system. This paper introduces the structure principle and operation characteristics of the UPFC, at the same time in Smiulink environment, the dynamic power quality in power system will be simulated to analysis. From the data results, it shows that UPFC can be better control the voltage and power, significantly improving the stability of power system, to prevent the system oscillation and the impact on power grid, so it has great practical significance.
\end{abstract}

\section{The basic structure of UPFC}

the structure diagram of UPFC system, as shown in figure 1.1, mainly including the main circuit and control circuit, the main circuit is composed of two systems which link to converter VSC1 through transformer T1 to parallel systems and converter VSC2 through transformer T2 series systems, the intermediate links is composed of DC power, its capacitance value is bigger, to ensure that the voltage fluctuation is not big,so under the condition of steady state operation, both ends of the capacitance $C$ is basic constant, its action is similar to a DC voltage; Control circuit is composed of control module and setting parameters, generally it used turn off thyristor control, from adjusting the different control Angle, respectively, controlling the separate serial-to-parallel converter output voltage, so as to achieve the purpose of regulating system of voltage and power.

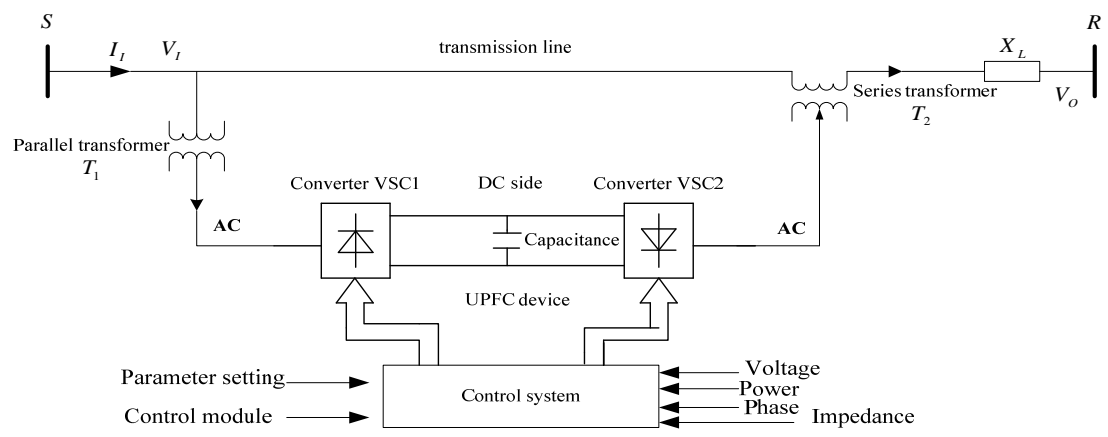

Figure 1.1 The structure diagram of the UPFC 


\section{The basic principle of UPFC}

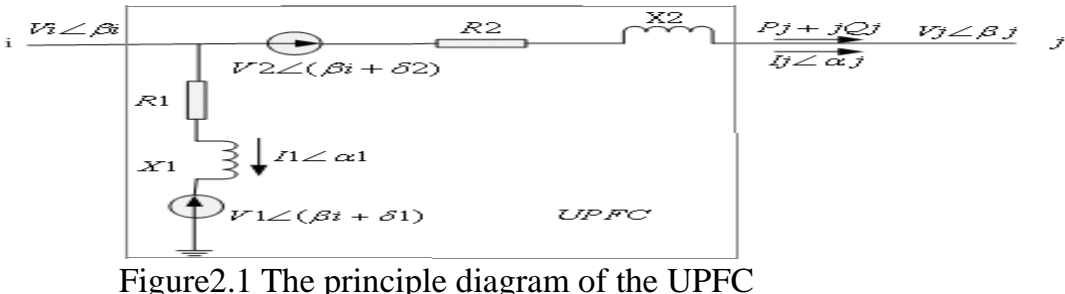

The mathematical model of UPFC equivalent circuit is shown in figure 2.1. from the figure, the converter VSC1 output voltage is $V_{1}$, the current is $I_{1} \angle \alpha_{1}$, voltage $V_{1}$ phase advance Angle to $V_{i}$ is $\delta_{1}$. the converter VSC2 output voltage is $V_{2}$, voltage $V_{2}$ phase advance Angle to $V_{i}$ is $\delta_{2}$.Based on UPFC instantaneous active power balance equation, getting the DC side capacitor C expressions of energy change:

$$
\frac{d\left(\frac{1}{2} C U_{d}{ }^{2}\right)}{d t}=\left[V_{1} I_{1} \cos \left(\delta_{1}+\beta_{i}-\alpha_{1}\right)-V_{2} I_{j} \cos \left(\delta_{2}+\beta_{i}-\alpha_{j}\right)\right]
$$

Derivation, the deformation of the expressions:

$\frac{d U_{d}}{d t}=\frac{1}{C U_{d}}\left[V_{1} I_{1} \cos \left(\delta_{1}+\beta_{i}-\alpha_{1}\right)-V_{2} I_{j} \cos \left(\delta_{2}+\beta_{i}-\alpha_{j}\right)\right]$

In the formula, $X_{1}$ - leakage impedance of parallel transformer ${ }^{T_{1}} ; X_{2}$ - leakage impedance of series transformer $T_{2} ; R_{1}$ - equivalent resistance of converter VSC1 and $T_{1} ; R_{2}$ - equivalent resistance of converter VSC2 and $T_{2}$.

If the system is stable, the capacitance rate for energy is $d u_{d} / d t=0$, so

$$
V_{1} I_{1} \cos \left(\delta_{1}+\beta_{1}-\alpha_{1}\right)=V_{2} I_{j} \cos \left(\delta_{2}+\beta_{i}-\alpha_{j}\right)
$$

From above the formula, if we do not consider the loss of converter VSC1 and ${ }^{T}$, it can be got parallel the current ${ }^{I_{1}}$ expression:

$$
I_{1} \angle \alpha_{1}=\frac{V_{i} \angle \beta_{i}-V_{1}\left(\beta_{i}+\delta_{1}\right)}{j X_{1}}
$$

Similarly, it can be introduced $V_{1}$ and $V_{2}$ as inverter output voltage for the dc voltage source, in order to better improving the fundamental component ,reducing the harmonics and improving power factor, SPWM technique can be used to control, it is concluded that the output voltage fundamental component is:

$V_{1}=k_{1} U_{d} \angle\left(\beta_{i}+\delta_{1}\right)$

$$
V_{2}=k_{2} U_{d} \angle\left(\beta_{i}+\delta_{2}\right)
$$

In the formula, $\delta_{1}$ and $\delta_{2}$ are the phase displacement of endpoint voltage waveform; $\quad k_{1}$ and $k_{2}$ are are the modulation factor

\section{The operating characteristics of the UPFC}

To illustrate the operation of the UPFC characteristics, presenting UPFC power transmission system diagram and the vector diagram schematic as shown in figure 3.1 ,Now it be supposed that the simplified equivalent reactance of transmission line is $X$.the send to the voltage vector is $\dot{U}_{s}$, and also the receive to the voltage vector is $\dot{U}_{r}$, respectively; $\delta$ is the Angle for the transmission line; $P_{s}, Q_{s}$ and $P_{r}, Q_{r}$ are active power and reactive power.with respectively sending and receiving. 


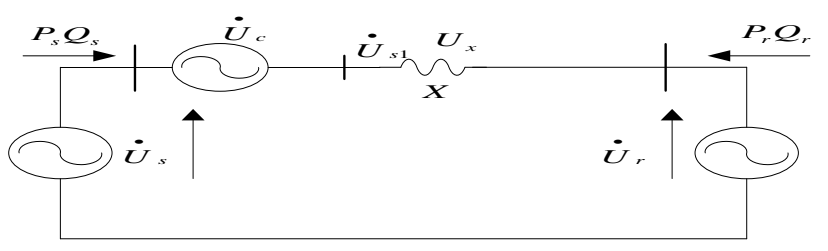

Figure 3.1(a) UPFC device in the transmission system

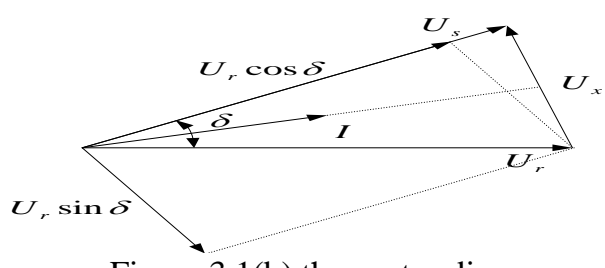

Figure 3.1(b) the vector diagram

From above the figure, take $\dot{U}_{s}$ as a reference vector, namely:

$$
\dot{U}_{s}=\dot{U}_{s} e^{j 0}=U_{s} \quad \dot{U}_{r}=U_{r} e^{-j \delta}=U_{r}(\cos \delta-j \sin \delta)
$$

So receive end of active power and reactive power can be calculated the following:

$P_{r}+j Q_{r}=\dot{U}_{r}\left(\frac{\dot{U}_{s}-\dot{U}_{r}}{j X}\right)$

Put formula (3-1) into formula (3-2) .namely:

$P_{r}+j Q_{r}=\dot{U}_{r}\left(\frac{\dot{U}_{s}-\dot{U}_{r}}{j X}\right)^{*}=U_{r}(\cos \delta-j \sin \delta)\left(\frac{U_{s}-U_{r} \cos \delta+U_{r} j \sin \delta}{j X}\right)^{*}$

Finishing formula:

$$
\begin{gathered}
P_{r}+j Q_{r}=\dot{U}_{r}\left(\frac{\dot{U}_{s}-\dot{U}_{r}}{j X}\right)^{*}=\frac{U_{r}}{X}(\cos \delta-j \sin \delta)\left(j U_{s}-j U_{r} \cos \delta+U_{r} \sin \delta\right) \\
=\frac{U_{r} U_{s} \sin \delta}{X}-j \frac{U_{r}\left(U_{r}-U_{s} \cos \delta\right)}{X}
\end{gathered}
$$

In the same way, deliver end of active power and reactive power can be calculated the following:

$$
P_{s}+j Q s=\dot{U} \cdot\left(\frac{\dot{U} s-\dot{U}_{r}}{j X}\right)^{*}
$$

At last finishing formula, namely:

$$
P_{s}+j Q_{s}=\dot{U}_{s}\left(\frac{\dot{U}_{s}-\dot{U}_{r}}{j X}\right)^{*}=\frac{U_{S}}{X}\left(j U_{s}-j U_{r} \cos \delta+U_{r} \sin \delta\right)=\frac{U_{r} U_{S} \sin \delta}{X}+j \frac{U_{s}\left(U_{s}-U_{r} \cos \delta\right)}{X}
$$

Comparison formula (3-4) and formula (3-6) , when ignoring the active loss of line, they are the same between receive end and deliver end of active power, namely:

$$
P=P_{s}=P_{r}=\frac{U_{r} U_{s} \sin \delta}{X}
$$

So two ends of reactive power can be expressed respectively as follows:

$$
\begin{aligned}
& Q_{r}=-\frac{U_{r}\left(U_{r}-U_{s} \cos \delta\right)}{X} \quad Q_{s}=\frac{U_{s}\left(U_{s}-U_{r} \cos \delta\right)}{X} \\
& \text { if } U_{s}=U_{r}=U \text {, so we can get: } \\
& P=P_{s}=P_{r}=\frac{U^{2} \sin \delta}{X} \quad Q_{s}=-Q_{r}=\frac{U^{2}(1-\cos \delta)}{X}
\end{aligned}
$$

and if $U_{r}=U_{s}=U=1, X=1$, at last we can get:

$$
P=P_{s}=P_{r}=\sin \delta \quad Q_{s}=-Q_{r}=(1-\cos \delta)
$$

According to the above formula, in order to adjust the line transmission of power , we can adjust the following parameters, such as the voltage amplitudes $U_{r}$ in the receive end, the voltage amplitudes $U_{s}$ in the deliver end, the line reactance $X$ and transmission line power angle $\delta$, if two ends of the voltage and line reactance value under constant conditions, we can change the Angle, so it has great significance for optimal operation of the system . 


\section{The influence of UPFC in power system dynamic power quality}

Dynamic power quality mainly includes the voltage drop, power oscillation and the influence of harmonic when the system is in failure. Joining up the UPFC device, it can significantly suppress the voltage drop, preventing the power oscillation, and providing the stability of the whole system.

\subsection{The establishment of simulation model of UPFC}

Now we set to both ends of the power supply, by a double line as distributed parameter transmission line, connected to the load of 300 MVA .Using the simulation MATLAB software, in the Smiulink environment, setting up the module related electrical parameters, at last it will be created UPFC simulation module, as shown in figure 4.1.

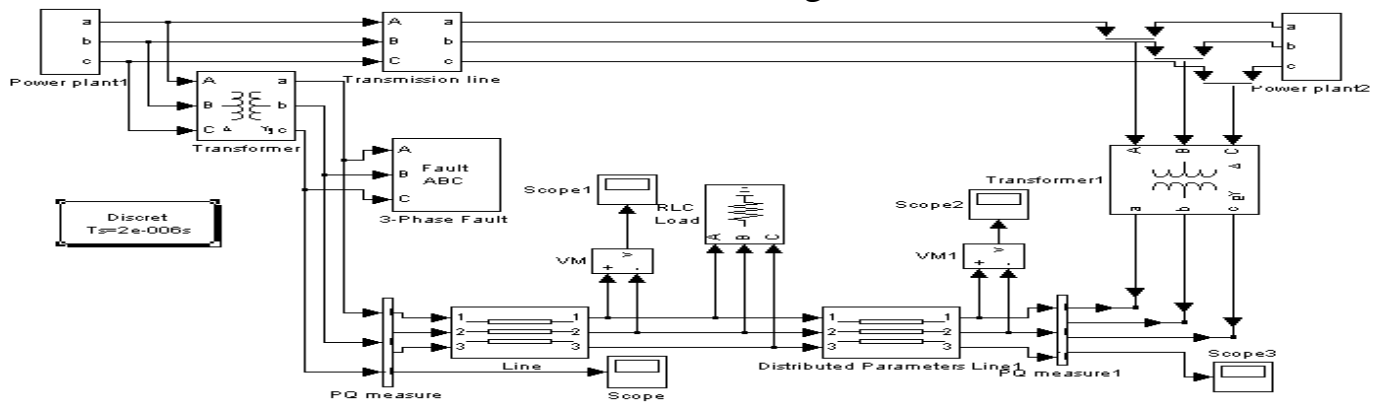

Figure 4.1 UPFC simulation module

\subsection{The influence of UPFC to power system voltage}

When power system failure, through parameter design, setting up the failure time of $0.01 \mathrm{~s}$ to $0.03 \mathrm{~s}$, as shown in figure 4.2, if it is not installed UPFC devices, the system voltage will be from the peak to damping, in the failure time, the voltage will be attenuation to 0 about $0.03 \mathrm{~s}$; but installation of the UPFC device, the system voltage won't appear obvious attenuation, just less amplitude will be reduced, but the waveform is close to sine wave.

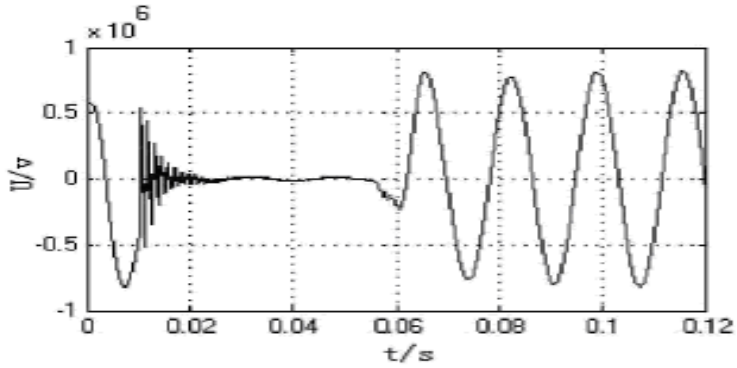

Figure 4.2 (a) the voltage waveform in system fault

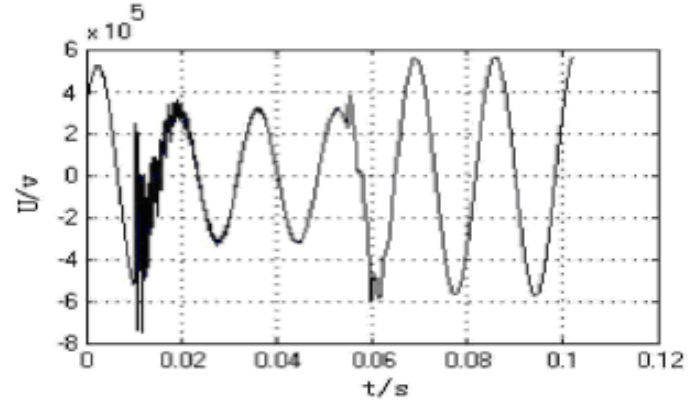

Figure 4.2 (b) the voltage waveform of installing the UPFC system

From above comparison, it shows that in the failure time during the installation of UPFC device in the system, the main circuit of transducer VSC2 through series transformer T2 changed part of the output voltage amplitude and reactive power output, so as to change the UPFC eventually reactive, and also it changes the system of voltage, but ensuring nature of the system voltage remains the same, so it improves the transient stability of the system.

\subsection{The influence of UPFC to power system power}

Through the parameter setting, power waveform can be as shown in figure 4.3, no installment UPFC devices, during the failure time from $0.01 \mathrm{~s}$ to $0.03 \mathrm{~s}$, the system power will be caused serious mutation, even if the failure resection, the system power still happen oscillation in a long time; but installation of the UPFC device, in the fault time, the power waveform does not occur larger mutation, system dynamic performance is for the better, after resection, the system power can be restored and stable operation in a cycle around. 


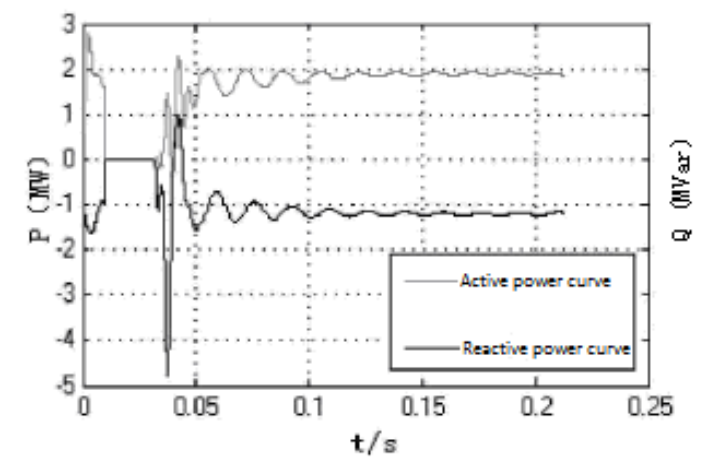

Figure 4.3 (a) the power waveform in system fault figure

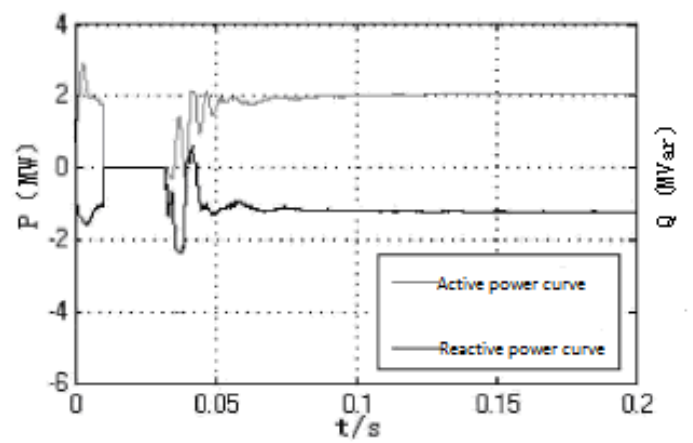

4.3 (b) the power waveform of installing the UPFC system

From above figure, installing UPFC device in the system can greatly restrain power oscillation, shortening the power oscillation period, and it also has obvious improvement effect on the stability of power system, at the same time, it is much more conducive to reducing the impact to the grid system.

\section{Conclusion}

This paper introduces the structure principle and operation characteristics of the UPFC, at the same time in Smiulink environment, it can be created a simulation model of UPFC, focusing on power system dynamic simulation analyses of power quality. From data results, it shows that the UPFC device can better realize the system voltage and power regulation, significantly improving the stability and security of the power system, preventing voltage drop and power system oscillation, and also reducing the impact on the grid, so it has great practical significance.

\section{References}

[1] Narain G. Hingorani\&Laszelo Gyugyi.,“Understanding FACTS, Concepts and Technology of Flexible AC Transmission Systems”.New York: IEEE Press and John Wiley\&Sons, Inc., 2000.

[2] A Nabavi Niaki, M R Iravani. Steady-state and dynamic models of unified power flow controller(UPFC) for power system studies. IEEE Transaction on Power Systems, 1996,11(4):1937-1943

[3]Predrag C Stefanov, Aleksandar M Stankovic. Modeling of UPFC operation under unbalanced conditions with dynamic phasors. IEEE Transaction on Power Systems, 2002,17(2):395—403

[4] wenjin Dai , ping Wang . Application of Pattern Recognition and Artificial Neural Network to Load Forecasting in Electric Power System. “IEEE ICNC'07 and FSKd'07 International Meeting”. 2007. 8 : 381 385. 